Correspondence: Georgina Jones, Department of Psychology, School of Social Sciences, Leeds Beckett University, Calverley Building, City Campus, LS1 9HE, Leeds, UK.

Tel.: 0113.8125106. E-mail: g.l.jones@leedsbeckett.ac.uk

Key words: Primary immunodeficiency; immunoglobulin treatment; qualitative research; thematic analysis.

Acknowledgements: We are extremely grateful to all the patients who gave their time to take part in this research and share their experiences of receiving Ig treatment. We are also extremely grateful to Professor John Brazier for his contribution to the development of the study protocol.

Contributions: GLJ: principal investigator for the study, wrote the study protocol for funding and ethics applications, co-wrote the interview schedule and assisted with data collection and analysis of the interview data. Wrote the paper for publication. EHB: supported analysis of the data and writing of the paper for publication. EH: contributed qualitative expertise to the writing of the paper for publication. RD: co-wrote the interview schedule, assisted with patient recruitment, undertook most of the qualitative interviews and assisted with analysis of the data. She assisted with the writing of the manuscript for publication. BG: contributed qualitative expertise to the methodology and writing of the paper for publication. JH: undertook some of the qualitative interviews and commented critically on the drafts of the manuscript for publication. DH: supported recruitment of the patients to the study and commented critically on the drafts of the manuscript for publication. FA: helped write the study protocol, assisted with recruitment of the patients to the study and contributed clinical expertise to the drafting of the paper for publication. JP: provided patient expertise, commented critically on the interview schedule, protocol and manuscript for publication. LS: provided patient expertise, commented critically on the interview schedule, protocol and manuscript for publication. JD: provided patient expertise, commented critically on the interview schedule, protocol and manuscript for publication. NM: provided clinical expertise, commented critically on the interview schedule, protocol and manuscript for publication. AS: helped write the study protocol, assisted with patient recruitment and contributed clinical expertise and interpretation to the drafting of the paper for publication.

Conflict of interest: $\mathrm{EH}, \mathrm{DH}, \mathrm{FA}, \mathrm{BG}$ and AS declare that they have no conflict of interest. GLJ has received a research grant from Baxalta US to undertake this work and received financial remuneration to attend two travel meetings to London as part of this study. EBH decares no conflict of interest. EBH is an employee of a contract research organization Parexel, but has received no financial rewards to participate in this publication. $\mathrm{RD}$ was a paid research associate working on this award and received financial remuneration to attend two travel meetings to London as part of this study. JH was also a paid research associate working on this award. JP and JD have previously received unrestricted grants from BAXALTA/SHIRE to support IPOPI's coordination work with its national member organisations in the context of the study. They have also received unrestricted grants from BAXALTA/Shire in support of IPOPI's activities, outside the scope of the study. NM has received unrestricted education grants from BAXALTA FRANCE and then SHIRE FRANCE to support the research activities of the French National Reference Center for PID (CEREDIH). He also received financial remuneration for travel to speak at the SHIRE International Symposium in 2017 and an honorarium for speaking at this symposium.

Conference presentation: The abstract of this paper was presented at the Advances in Patient Reported Outcomes: Integration and Innovation Conference, Leeds, UK, $13^{\text {th }}$ June 2019 as an oral presentation with interim findings. The abstract has been published in the Journal of Patient-Reported Outcomes 2020, 4 (Suppl 1):28.

Funding: This work was supported by Baxalta US (now known as Takeda) under grant number AM000958.

Received for publication: 16 December 2020

Accepted for publication: 7 January 2021.

This work is licensed under a Creative Commons Attribution NonCommercial 4.0 License (CC BY-NC 4.0).

${ }^{\circ}$ Copyright: the Author(s), 2020

Licensee PAGEPress, Italy

Qualitative Research in Medicine \& Healthcare 2020; 4:117-131

doi:10.4081/qrmh.2020.9564

\section{It's long-term, well it's for life basically: Understanding and exploring the burden of immunoglobulin treatment in patients with primary immunodeficiency disorders}

\author{
Georgina L Jones, ${ }^{1}$ Eva Brown Hajdukova, ${ }^{2}$ \\ Esmee Hanna, ${ }^{3}$ Rosie Duncan, ${ }^{4}$ Brendan Gough, ${ }^{1}$ \\ Jane Hughes, ${ }^{4}$ Debbie Hughes, ${ }^{5}$ Fran Ashworth, ${ }^{5}$ \\ Johan Prevot, ${ }^{6}$ Jose Drabwell, ${ }^{6}$ Leire Solis, ${ }^{6}$ \\ Nizar Mahlaoui, ${ }^{7}$ Anna Shrimpton ${ }^{5}$ \\ on behalf of the International IgBoT study team
}

${ }^{1}$ Department of Psychology, School of Social Sciences, Leeds Beckett University, Leeds; ${ }^{2}$ Parexel Access Consulting, Parexel International, London; ${ }^{3}$ Allied Health Sciences Research, De Montfort University, Leicester; ${ }^{4}$ School of Health and Related Research, University of Sheffield, Sheffield; ${ }^{5}$ Clinical Immunology and Allergy Unit, Northern General Hospital, Sheffield Teaching Hospitals and NHS Foundation Trust, Sheffield; ${ }^{6}$ International Patient Organisation for Primary Immunodeficiencies, Rocky Bottom, Trerieve, Downderry, Cornwall, United Kingdom; ${ }^{7}$ French National Reference Center for Primary Immune Deficiencies (CEREDIH), Necker Enfants Malades University Hospital, Assistance Publique-Hôpitaux de Paris, Paris, France; Pediatric Immuno-Haematology and Rheumatology Unit, Necker Enfants Malades University Hospital, Assistance Publique-Hôpitaux de Paris, Paris, France 


\section{Introduction}

Primary Immunodeficiency Disorders (PIDs) consist of a group of rare disorders characterised by an impairment in the development and maturation of the immune system. ${ }^{1}$ This means that for people affected by PIDs, parts of their immune system are missing or not working properly leaving them with a reduced or absent natural defence against viruses, bacteria or fungi. The consequences of this immune deficiency are that many people with PIDs will experience frequent infections which do not resolve as quickly as would be expected with antibiotic treatment and even then, the infections may keep recurring. ${ }^{1,2}$ These symptoms can be mild e.g. recurrent colds or severe illnesses including pneumonia, skin disorders, arthritis and heart problems to name a few. ${ }^{3}$ As such, the symptom and disease burden associated with PIDs often have a significant negative impact upon a patient's quality of life. ${ }^{4}$ Children and adults can experience frequent absenteeism from school or work, reduced participation in social and sporting activities, as well as a range of psychological symptoms in response to living and coping with their condition. ${ }^{2}$

Immunoglobulin (Ig) treatment is the main therapy for most patients with PID and it is typically administered either intravenously (directly into a vein) or subcutaneously (directly under the skin). Whilst it is generally accepted that this treatment can dramatically improve quality of life, $, 1,5$ it may also be associated with a substantial treatment burden i.e. work and effort for the patient. For example, intravenous Ig treatment (IVIg) is typically administered in hospital (although it can also be given at home in some circumstances). It is usually infused every 3 weeks but can range from 2 to 4 weeks lasting approximately 2-4 hours per visit. The precise length of infusion however, will be dependent on dose and tolerance of the individual. Side effects include headache, feeling hot, nausea, diarrhoea, rash, back pain, rarely hypotension. Extremely rare and serious side effects such as allergic reactions, kidney problems or blood clots. Subcutaneous Immunoglobulin (SCIg) infusions are given by slowly injecting immunoglobulin into fatty tissue just underneath the skin. SCIg can be given at home, using a mechanical infusion pump (spring loaded, or battery powered) or by rapid push (a manual method that does not require a pump, infusion is pushed by hand through a syringe). The use of the latter method has been increasing over time. Common side effects to SCIg include site reactions such as redness, swelling and itching which are usually mild and go away over a day or two. Subcutaneous Ig treatment (SCIg) is typically administered 1-3 times per week in the home but can be administered in the hospital depending on patients' individual needs, but this is generally considered the exception rather than the rule. ${ }^{6}$

The 'work of being a patient' is referred to as burden of treatment - everything the patient needs to do to treat and manage their illness, for example undergoing tests and investigations, visiting doctors, adhering to treatment regimens and making lifestyle changes. ${ }^{7}$ Burden of treatment is a concept independent of the disease burden and can be defined as the consequences of receiving treatment (these may be medication, therapies or other interventions) ${ }^{8-10}$ It is an important concept because it may negatively affect adherence to treatment, quality of life, disease management and health care outcomes such as hospitalisations and survival. ${ }^{11,12}$

Whilst there are numerous studies which have tried to measure the quality of life of patients with a PID, ${ }^{1,5,13-22}$ less attention has focused upon the burden of Ig treatment. A recent systematic review which synthesised the evidence regarding the potential burden of Ig treatment found that overall, PID patients reported little Ig treatment burden and appeared satisfied with IVIg and SCIg therapy. ${ }^{23}$ The patients' preference appeared to be the delivery of the Ig treatment in the patient's home and SCIg was preferred after switching from IVIg therapy. However, most studies had adopted a quantitative approach using questionnaires and there was a lack of qualitative research which, when applied in a health service setting, has the potential to gain a richer and deeper insight into the patient's lived experience with an illness or treatment - particularly if the condition is chronic in nature. ${ }^{24}$ For this reason, the lack of qualitative research in this area was considered an important gap in the literature. One recent mixed-method study undertook a qualitative exploration of Facilitated Subcutaneous Immunoglobulin (fSCIg) to explore treatment burden and analysed patient's responses to open-ended questions using a thematic analysis. However, the study included patients with both a primary and secondary immunodeficiency receiving fSCIG, and unfortunately did not report the findings separately for these different patient groups. Nevertheless, these patients described experiencing some immediate post treatment symptoms such as fatigue, and difficulties using the infusion equipment. But, overall low treatment burden was reported due to the time-saving nature of the treatment and overall improvements to health and well-being that occurred, thus outweighing any negative aspects associated with the treatment. ${ }^{25}$

\footnotetext{
Aims

Therefore, the aims of this paper are to describe the results of a qualitative, cross-sectional study carried out to explore in-depth the burden of treatment i.e. the 'work of being a patient', for patients diagnosed with a PID and undergoing Ig treatment. To the best of our knowledge, this is the first study which has solely sought to provide a rich insight into the potential burden of Ig treatment for this patient group. As the aim was to understand the patient's lived experience of Ig treatment burden, we used a phenomenological theoretical approach to guide the interpretation of the data.
} 


\section{Materials and Methods}

\section{Study design}

In order to gain an in-depth understanding of the burden of Ig treatment for patients with a PID we adopted a qualitative methodology. We conducted thirty semi-structured interviews with patients receiving intravenous $(\mathrm{n}=21)$ and subcutaneous immunoglobulin $(\mathrm{n}=9)$ therapy, either at home or in hospital and we approached patients attending the Clinical Immunology and Allergy unit (CIAU) at a hospital in Yorkshire, UK between March 2015 to October 2015 to participate in the study. Ethics approval for our study was granted from the NRES committee Yorkshire and the Humber - Bradford Leeds on $31^{\text {st }}$ October 2014. The REC reference was $14 / \mathrm{YH} / 1247$.

\section{Participant selection}

Our inclusion criteria were that patients aged 16 years and older, who had been diagnosed with a PID and were undergoing Ig treatment (either SC, IV at home or in hospital). We excluded patients if they were receiving Ig treatment for a secondary immunodeficiency e.g. secondary to lymphoproliferative disease or following treatment with immunosuppression or if their medical condition meant that they were unable to undertake an interview.

\section{Participant recruitment}

Our members of the clinical care team (FA, AS and DH) identified patients from the PID patient database held at the Clinical Immunology and Allergy Unit. They posted the patient information sheets and a consent form to potential participants with a pre-paid addressed envelope to return to the clinic if they were interested in taking part. The clinic team then passed onto the researcher the details of those who had returned the completed consent form. We asked patients whether they had received the posted information when they attended for appointments. We did not provide incentives to patients to participate in the study, but we did reimburse their travel and/or parking costs. Our researchers (RD or JH) then visited the patient in the hospital on their infusion date if they were hospital - based or at home to undertake the interviews.

\section{Interview guide development}

We developed a semi-structured interview schedule to help researchers guide the conversation and keep respondents on topic as well as to encourage two-way communication. Our semi-structured interview schedule also provided an opportunity for the interviewers to capture not only the answers to questions, but also learn about the reasons behind the answers, as well as allowing respondents time to open up about sensitive issues. We initially generated the questions for the interview schedule following i) a meeting with some members of the team which involved academic, clinical and patient advocates, and ii) by the emerging results of a systematic review. Some members of our team were undertaking this in parallel to the qualitative study, which had started to identify the areas of Ig treatment burden for patients with a PID from the international literature. ${ }^{24}$ Our initial schedule was then circulated to the wider team for final internal peer review and feedback following which minor amendments were made.

\section{Data collection process}

We used a purposive sampling method whereby patients were approached for participation in the study to ensure maximum diversity within the sample, to reflect gender, a range of age groups, a variety of PID conditions, length from diagnosis of PID, length of time on treatment and types and modes of treatment (i.e. infusing at home or hospital). We anticipated that interviews with 25-30 patients may be needed to reach data saturation, although, we agreed that recruitment would continue until thematic saturation was achieved.

In broad terms, saturation is used in qualitative research as a standard for discontinuing data collection and/or analysis. Saturation guides acceptance across a range of approaches to qualitative research and is often proposed as an essential methodological element within such work. Failure to reach saturation has an impact on the quality of the research conducted and it is considered to be the gold standard by which purposive sample sizes are determined in health science research. ${ }^{26}$ Saturation is commonly described as a rule of qualitative research, and it features in a number of generic quality criteria for qualitative methods. The saturation model used in this study is aligned with the data analysis approach as it is focused on the identification of new themes. More specifically, it is based on the number of such themes rather than the completeness of existing theoretical categories. This approach is termed inductive thematic saturation and can be described as extent to which 'new' codes or themes are identified within the data, and/or the extent to which new theoretical insights are gained from the data via this process. $^{26}$

It has been established in the qualitative methods literature that saturation can be reached with sample size as small as 12 participants. ${ }^{26}$ However, in our study saturation was reached by the $30^{\text {th }}$ interview as it was agreed by the data analysts that the inclusion of additional study participants did not provide any substantially new or previously unrecognized issues or concepts. Our data analyst team members carefully monitored the point when saturation was achieved throughout the qualitative data analyses by populating a tabular summary known as a saturation grid.

Two of our researchers undertook the interviews (RD and $\mathrm{JH}$ ). $\mathrm{RD}$ is a psychologist and $\mathrm{JH}$ is an experienced health service researcher. They had both received training in qualitative research methodology. Patients receiving 
their treatment in hospital were offered the option of being seen at home if they preferred.

Considerable efforts were undertaken by us to establish the research's trustworthiness as defined by Lincoln and Guba. ${ }^{27}$ For example, we have presented rich quotes to support the themes derived in order to support findings in other contexts (transferability). In addition, we kept a detailed audit trail of the data generated and used as part of the study (e.g. the interview transcripts, audio recordings and coding framework and system) which several members of our research team reviewed (dependability). It could be argued that the confirmability (objectivity) of the research findings may be compromised as the funder of the research is a manufacturer of Ig products. However, the funder of our research did not have any involvement in the data collection and analysis process and the transcriptions and audio recordings were not shared with these team members. The members of our study team that were specifically involved in the data collection and analysis (GJ, RD, JH and $\mathrm{EH}$ ) were from academic institutions and were not responsible for the clinical treatment and management and/or personal care of any of the patients that took part.

We recorded the interviews using the Olympus DS700 digital voice recorder and transcribed these verbatim (names and identifiable information were not included in the transcripts). An independent transcriber (not part of the study team) undertook the transcriptions. Members of our research team checked the quality of these and assisted the transcriber where clarification was needed (e.g. the sound was too quiet, and/or unfamiliar clinical words were used). We uploaded the transcripts into NVivo version 10 for data management and analysis.

\section{Data analysis}

Qualitative data analysis is a broad term encompassing different analytical approaches with the aim of providing contextual description and interpretation of social phenomenon. ${ }^{28}$ Qualitative content analysis (QCA) and thematic analysis (TA) are both classified under the qualitative descriptive design and are widely used by qualitative researchers. TA are similar to QCA in terms of philosophical backgrounds, immersion in data, attention to both description and interpretation of data analysis, consideration of context during data analysis, and cutting across data for seeking themes. As a point of difference, within TA, a theme is considered to be latent content, but QCA researchers are free to decide between the level of data analysis when developing the category or the theme. Nevertheless, to develop a theme in both of these approaches, iterative or forward-backward movements and comparison of code clusters in relation to the whole data are required. ${ }^{28,29}$

Researchers using QCA may prefer to work with simplicities and overt data by going through a large amount of text to obtain easy-achieved classifications and manifest contents to develop categories. On the other hand, those using TA require an exhaustive and non-stop process of abstraction and in-depth analysis from the beginning to reach the theme. ${ }^{28}$ It is believed that high-quality qualitative research is marked by a thick description, and rich complexity of findings rather than deductive precision. The complexity and aesthetic aspects of the theme can motivate readers to reflect on data and relate the findings to their own personal perspectives. Analysing data qualitatively and also quantifying data are possible in QCA, but in TA a purely qualitative account of data is utilized. ${ }^{29}$ Based on the description above, we adopted an inductive [data-driven] thematic analysis approach to prioritise patient accounts/concerns and identify the key concepts and themes. We also selected the TA approach because it is likely to enable for production of more trustworthy and insightful findings by providing a rich and detailed, yet complex account of the patient data. ${ }^{28}$

Friese reports that using qualitative software to analyse data does not diminish the quality of the qualitative research nor does it simplify the whole process of doing qualitative research. When used correctly, it can actually ease the data management and data analysis processes and facilitate analytic rigor. ${ }^{30}$ As NVivo provides a valuable tool for analysing the interviews thematically, NVivo version 10 was used for both effective data management and analysis. However, it should be noted that NVivo software can only help the researcher to manage, explore and find patterns in data but it cannot replace the analytical expertise.

In the data-driven thematic analysis approach adopted in this study, the categories (codes) were selected based on a detailed analysis of all data. This approach was particularly suitable as the goal was to make an in-depth exploration of the data. Coding of the data into the categories of analysis required the execution of an explicit set of recording instructions about the rules for coding the data into categories. This process involved more than one judge as the process of triangulation common in qualitative research is designed to enhance the quality of and confidence in the analysis. ${ }^{28}$

More specifically, four transcripts were read independently by two researchers (RD and GJ) to produce an initial coding framework. A meeting was held to discuss and agree the modifications to the framework. One researcher (RD) then went through the other 26 transcripts and coded using this framework, also adding new codes as they arose. Units of text that clustered together were then grouped into analytic categories - themes. It is important to note that, if appropriate, the same unit of text was included in more than one category. The data were then systematically revisited to ensure that a named theme had all data relevant to support the topic included.

Given the under-researched nature of the topic, and specifically of the presentation of the voices of those undergoing Ig therapy, we have chosen to include rich personal testimony in the form of quotations within the 
results section in order to ensure that the voices of a group we rarely hear from, are in this context, heard.

\section{Results}

We sent fifty-two PID patients a participant information sheet and consent form, of whom 35 (68\%) consented to take part in the study. In total, we conducted 30 semistructured interviews either in patient's homes or at the CIAU between March 2015 and October 2015. We stopped interviewing at this point, because data saturation was reached and therefore the remaining five patients were not interviewed. We present the clinical characteristics of the patient sample in Table 1 and a summary of their demographics in Table 2.

\section{Burden of Ig treatment themes}

In the effort to undergo and maintain Ig infusions and care for their health, we generated eight themes to describe patients' experiences of treatment: i) time burden; ii) treatment consequences; iii) impact on social interactions and relationships; iv) treatment environment; v) affects emotional well-being; vi) travel; vii) role functioning; and viii) financial consequences. For each patient quote we report the patient's unique study number $=\mathrm{IDx}$, the mode and place of treatment $=\mathrm{IV} / \mathrm{SC}$, home/hospital, and their gender $=\mathrm{M} / \mathrm{F}$.

\section{i) Time burden}

In this theme, we describe how Ig treatment negatively impacted upon patients' time and was the main burden of
Ig treatment described by patients. Embedded within this theme were three subthemes including: i) It takes up too much time; ii) Time management - planning and organising routines; and ii) Inconvenience/arranging timetables around others.

\section{It takes up too much time}

A considerable amount of time and commitment was required in order to undergo this treatment regardless of the mode of Ig therapy and location (home or hospital). The amount of time varied by mode and location of Ig treatment administration. Generally, patients described having to take at least a couple of hours to a day every few weeks to receive treatment. For people taking SCIg, treatment was administered more regularly than for people taking IVIg treatment. For those on the highest dose of SCIg this meant infusing twice a week. As well as the weekly time burden, many of the patients had been infusing over a number of years which exacerbated the time burden:

"I think it's just because I've been doing it for like $32 / 33$ years, and that's quite a big commitment isn't it, so that for me at times, I just get every three weeks thinking 'argh here we go again', and I really wish I could have like a break, and not have to do it this time, but I know I can't, so I do." (ID24 IV Home/ M).

In addition, some patients felt that SCIg home therapy was preferable to hospital treatment as it didn't take up as much time, as one participant described:

Table 1. Clinical characteristics of the patient sample.

\begin{tabular}{llc}
\hline Patient characteristics & & N (\%) \\
\hline Mean age: & & 52.9 years (SD: 17.5, range: 27-92 years). \\
\hline Gender: & Male & $13(43.3)$ \\
& Female & $17(56.7)$ \\
\hline Treatment mode: & IVIg home & $5(16.7)$ \\
& IVIg hospital & $16(53.3)$ \\
& SCIg home & $8(26.7)$ \\
& SCIg hospital & $1(3.3)$ \\
\hline Diagnosis: & Common variable immune deficiency & $20(66.6)$ \\
& Specific antibody deficiency & $3(10)$ \\
& Undefined antibody deficiency & $2(6.6)$ \\
& Hypogammaglobulinemia & $2(6.6)$ \\
& X-linked agammaglobulinemia & $1(3.3)$ \\
& Selective IgA deficiency* & $1(3.3)$ \\
& CD19 deficiency & $1(3.3)$ \\
\hline Duration of treatment: & <1 year & $3(10)$ \\
& 1-5 years & $10(33.3)$ \\
& 6-10 years & $5(16.7)$ \\
& $>10$ years & $12(40)$ \\
\hline
\end{tabular}

*NB: Whilst the diagnosis of selective IgA deficiency is not an indication for immunoglobulin replacement therapy per se, this patient had recurrent sinus and ear infection with end organ damage and chronic rhinosinusitis/conjunctivitis. A few patients with isolated IgA deficiency behave like panhypogammaglobulinaemia cases (i.e. more severe antibody deficiency') and require immunoglobulin. Therefore, the multidisciplinary team at the CIAU recommended that the patient start on immunoglobulin replacement therapy and hence they were included in this study. 
"It's took a lot less time, not having to go to hospital. You haven't got to waste a day, or waste half a day in the hospital, wasting doctor and nurses time... but now I just go to work, come home one day, have my tea, and then start this treatment straight afterwards.” (ID35, IV home/M)

The way in which time was experienced in terms of 'waiting' or 'wasting time' in the hospital setting is in the quote above, therefore juxtaposed against the relative freedom and lack of constraints felt when the treatment was at home, 'in their own time'.
Time management - planning and organising routines

Generally, other issues related to time were about the need to plan and organise their own time effectively which required considerable effort on behalf of the patient. $\mathrm{Pa}-$ tients' often described having to keep to a strict routine to manage their treatment and frequently plan ahead, regardless of what mode of administration they were receiving:

"So I prepare- I've got this nice little table that I use, and it's easy to sterile and everything. I get my delivery every three months. I make packs up.

Table 2. Summary of the study participants demographics.

\begin{tabular}{|c|c|c|c|c|c|}
\hline Interview number & Participant ID & Male or Female & Age at time of interview & $\begin{array}{l}\text { Treatment at home } \\
\text { or in hospital }\end{array}$ & IV or SC \\
\hline 1 & ID01 & M & 42 & Hospital & IV \\
\hline 2 & ID02 & M & 27 & Hospital & IV \\
\hline 3 & ID03 & $\mathrm{F}$ & 55 & Home & $\mathrm{SC}$ \\
\hline 4 & ID04 & M & 52 & Hospital & IV \\
\hline 5 & ID06 & M & 70 & Hospital & IV \\
\hline 6 & ID09 & M & 77 & Hospital & IV \\
\hline 7 & ID11 & M & 36 & Hospital & IV \\
\hline 8 & ID12 & M & 34 & Hospital & IV \\
\hline 9 & ID13 & M & 29 & Home & IV \\
\hline 10 & ID14 & $\mathrm{F}$ & 58 & Hospital & IV \\
\hline 11 & ID15 & $\mathrm{F}$ & 57 & Hospital & IV \\
\hline 12 & ID18 & M & 92 & Hospital & $\mathrm{SC}$ \\
\hline 13 & ID19 & F & 80 & Hospital & IV \\
\hline 14 & ID20 & $\mathrm{F}$ & 83 & Hospital & IV \\
\hline 15 & ID22 & $\mathrm{F}$ & 83 & Hospital & IV \\
\hline 16 & ID23 & $\mathrm{F}$ & 62 & Hospital & IV \\
\hline 17 & ID24 & M & 47 & Home & IV \\
\hline 18 & ID28 & $\mathrm{F}$ & 43 & Home & IV \\
\hline 19 & ID29 & $\mathrm{F}$ & 52 & Home & $\mathrm{SC}$ \\
\hline 20 & ID33 & $\mathrm{F}$ & 32 & Home & $\mathrm{SC}$ \\
\hline 21 & ID35 & M & 52 & Home & IV \\
\hline 22 & ID36 & $\mathrm{F}$ & 54 & Home & $\mathrm{SC}$ \\
\hline 23 & ID39 & F & 43 & Home & $\mathrm{SC}$ \\
\hline 24 & ID40 & $\mathrm{F}$ & 53 & Home & $\mathrm{SC}$ \\
\hline 25 & ID41 & $\mathrm{F}$ & 50 & Home & $\mathrm{SC}$ \\
\hline 26 & ID43 & $\mathrm{F}$ & 51 & Home & IV \\
\hline 27 & ID46 & M & 41 & Hospital & IV \\
\hline 28 & ID47 & $\mathrm{F}$ & 67 & Hospital & IV \\
\hline 29 & ID49 & $\mathrm{F}$ & 33 & Home & $\mathrm{SC}$ \\
\hline 30 & ID51 & M & 33 & Hospital & IV \\
\hline
\end{tabular}


So everything I need for that week, so I make me 12 packs up ready." (ID 36 SC Home/F).

For some, their routines involved their partners and family members and treatment was woven into the structure and routines of their everyday lives, including fitting around meals and other activities:

"So like today he's in Manchester. So as long as he doesn't get stuck in traffic, half an hour before he's due home, he'll ring and say 'I'll be home in half an hour, take your treatment out the fridge'. By the time he's come in and you know we've had a cup of tea, it's about room temperature. Connect it up, and then what I do while it's in my tummy, I pop the machines in a handbag, put it over my shoulder and I can still do tea" (ID33 SC Home/F).

Emotionally, some participants found that the lack of flexibility concerning when they had their Ig treatment got them down at times. This was regardless of whether people were having IVIg or SCIg treatment and the location they were having the treatment. For example, one participant explained:

"I think I've got to that point where I know I've got to have it, and I can't escape it. Whereas I've tried before, I know I can't now, I think I've come to terms with it" (ID12 IV hospital/M).

Another participant felt that receiving Ig treatment in hospital was preferable given the additional pressure of having to keep their home environment ready and suitable for the treatment:

'It's easier if you know you're coming to hospital, you know you're going to have half a day. You just turn up, you sit, you're done, you go home. You don't have to worry about it. Whereas at home you're obviously, you're in your home environment, various things come up. You've obviously got to try and keep it consistent, you know, infusion day, you know make sure obviously your products out the fridge, make sure all your supplies are there, it's just more to think about. For me, this is easier" (ID51, IV hospital/M).

\section{Inconvenience/arranging timetables around others}

Those patients receiving SCIg at home who rely on an infusion partner also described the difficulty of fitting their SCIg at home in with the other person's schedule and having to rely upon others to help facilitate treatment, because as one participant reported: "You can't infuse while you're on your own. So, what we do is we just sort of work it round [husbands name] work timetable" (ID33, SCIg home F).

Another participant said:
"I childmind for my youngest daughter Monday, Tuesday, Wednesday evenings only for an hour. And it's not the treatment's fault. If transport is late, I don't get home on time to go and childmind. So now they say, 'oh is it your treatment week mum?' And I say, 'yes it's my treatment week" (ID23 IV Hospital/F).

Therefore, some participants, and their families were having to alter plans and make allowances for the disruption (or potential) disruption that treatment may bring. Sometimes this specifically related to the act of treatment itself, but also the experience of accessing the hospital and specifically the need for hospital organized transport which created knock on effects or inconveniences in their own as well as their family's lives.

\section{Treatment consequences}

This theme describes the ways that undergoing Ig treatment impacted upon the physical health of patients. A wide range of experiences with the medication was expressed by over half of the patients. Within this theme were four key subthemes including: i) impact upon skin, ii) side effects, and iii) tiredness and fatigue.

\section{Impact on skin}

Many of these skin-related impacts arose from the use of needles. In this respect, one female on subcutaneous treatment shared that: "My veins aren't very good, and they had become scarred and it was getting harder and harder to get cannula's in" (ID39 SC Home/F).

Some patients reported that the treatment itself was uncomfortable - whereas others described more specific side effects such as sore sites from the infusion, bruising, swelling and tightness. A couple of participants described not liking the look of their infusion site due to the swelling from treatment. Some patients who had been undergoing Ig treatment for a long time described having scarring on their veins:

"I get everything prepared and I do it, and it does help, visualising it in that way. I hate afterwards when I've finished, the site area is really sore, it's swollen and because I've got a scar from my belly button down" (ID 36 SC Home/F).

\section{Side effects}

Some, although not all, experienced other side effects or what they described as a 'reaction' to the medication which required additional steroid and/or antihistamine injections, for example fainting, skin reactions (itching, rashes, boils) and an allergic reaction:

"Red spots, well not spots, but boils everywhere. Small boils everywhere. All my body I feel to scratch my body. And at the time, I was already 
out, so I ran back here. They gave me a Piriton, to sit down and relax and then it disappeared" (ID 46 IV Hospital/M).

Not all reactions were in terms of welts or itching, some reported temperature issues after finishing treatment and the need to try and remedy this before departing from treatment,

"For a period of time, I did suffer really bad with shivers. When I'd finished my treatment, I would literally be like dithering, and I'd be sat in the car for a good 20 minutes/ half an hour, with the engine on and the heater blowing. I mentioned it to the nurses, and they were a bit concerned because they thought it could be an early stage of a reaction" (ID01 IV Hospital/M).

Side effects were therefore experienced in different ways by patients, but the concern about it being a problematic reaction to the treatment itself was common within these experiences.

\section{Tiredness and fatigue}

Many patients also described feeling tired and/or lethargic immediately after the treatment. One person described feeling so tired after treatment that they could not help but sleep for a number of hours post treatment. Fatigue was therefore for some an unpleasant side effect of treatment:

"I don't like, I don't like that feeling that I know in about half an hour to an hour's time, I've got to go to sleep. And I think when your body does that and you haven't got a choice, it isn't a nice feeling." (ID 36 SC Home/F).

Overall, patients described feeling tired regardless of their treatment mode. However, for a couple of patients the changes in energy levels appeared less of a problem with SCIg treatment compared with IV.

\section{Impact on social interactions and relationships}

Undergoing Ig treatment impacted upon the social life and personal relationships of many of the patients and was another core theme generated from the analysis. The ways this was experienced is described under three sub-themes including i) impact upon holidays and travel, ii) missing out on social activities, and iii) affecting family relationships.

\section{Impact on holidays/travelling}

Having to take treatment regularly and take this time out meant re-arranging leisure or social activities such as going on holiday. In addition, as Ig treatment should be taken every few weeks, patients frequently talked of having to plan their treatment around holidays:
"If you went away for three weeks, you'd be ok, but I think if it was any longer than that, so I don't think I can go on a round the world cruise without making some special arrangements" (ID06 IV Hospital/M).

Time was therefore seen as being constrained by the regularity of treatment and the perceived 'maximum' time that patients could be away for on holiday before needing to make additional provisions to ensure they were able to access treatment in a different way or place.

\section{Missing out on social activities}

Some described the Ig routines and disruption as being frustrating and getting them down because they missed out on other social activities they would prefer to do:

"I suppose on the back of that, the other impact it had is things like social life and feeling normal alongside your friends, because I'd go into hospital once a week, once a fortnight, and probably spend 2 or 3 days recovering from that, just getting to feel well enough again” (ID24 IV Home/M).

However, some patients did say the treatment itself did not interfere with their social life, but that they may have to adapt the type of holidays or activities they did in order to accommodate treatment.

\section{Affecting family relationships}

Tensions between family members developed, typically because family members tried to ensure that their loved ones continued taking their Ig treatment:

"Sometimes it frustrates me, and other times I just get on with it and do it. It does cause a lot of arguments... if I forget, he's (partner) like 'you need to do you treatment!" (ID49, SC Home/F).

Others felt that treatment impacted on their familial relationships as it caused their family members to worry about them more than they might have done had the patient not been receiving Ig treatment:

\footnotetext{
"Because their dad died when they were quite young, they've always been really worried then if I was poorly, so then that was another dimension that was particularly hard, because I would try not to let them know so they didn't worry, and you know, that sort of thing" (ID41 SC Home/F).
}

\section{Treatment environment}

The place where patients had their Ig treatment added to the burden, particularly for patients on SCIg at home. The burden of home treatment related to self-care activi- 
ties such as the effort of finding a suitable place to store the items needed for treatment as well as keeping the treatment area sterile and clean. The reasons for this was explained by one woman on SCIg who said: "Because you can't put your bottles in the same fridge as a normal fridge, as your food. So, I have a separate fridge, so that's very good (ID40 SC Home/F).

These specific requirements may therefore be a burden for some, in terms of having the space and resources to have an additional fridge or space they could keep and make sterile for their home treatment.

Some of the participants taking home therapy reported missing the social contact provided by going into clinic:

"I used to go a day a week into hospital and then eventually I was on home therapy and that were fantastic. I do miss, miss them though. I said to [name deleted] because I used to go in one day a week, and you get so looked after, and because you're chatting to other patients and other people, and you're watching all the people, and when you go on home therapy you do lose that" (ID $36 \mathrm{SC}$ Home/F).

\section{Emotional burden}

There appeared to be a psychological burden associated with undergoing Ig treatment which this theme describes. It has four sub-themes including: i) feeling powerless and depressed, ii) lack of flexibility, iii) fear about self-injecting and using blood products, and iv) life revolves around the treatment.

\section{Feeling powerless and depressed}

Emotions ranged from feeling apprehensive about the treatment to feeling worried and depressed,

"When it was coming around to treatment, it did used to depress me like because I know that when I get it I'm going to be here for like 5 hours or something like that, and it's tiring, sat in hospitals" (ID12 IV Hospital/M):

Others noted feeling powerless in the face of the treatment they were undergoing and the challenges of managing that treatment with work and other commitments:

"Because it was depressing me that much, you know I need to come all time, so I just kept, and I were working as well. I had to keep having days off work and stuff like that, and it was getting me right down like. So, I just thought 'stuff it', I weren't bothered, and I probably went a few months, I've done it a few times you know" (ID12 IV Hospital/M).
“... it takes out a day and a half of your week, and it has an impact then on lots of things. If you think of your week, if you missed out on 36 hours of your week, I think you'd be, it's hard to, something has to give doesn't it, in that way. Sometimes I can get a bit, a bit miffed about it" (ID36 SC Home/F).

\section{Fear about self-injecting/using blood products}

A number of people discussed having a dislike of needles and finding the injection and infusion process unpleasant both to go through and look at. One patient, despite having had Ig treatment for a while, described that he: “... can't get used to it no, never. I can't look at it, you know when they're putting it in, I can't look at it at all" (ID12 IV hospital/M).

Another male participant undergoing IVIg treatment at home recalled the amount of time it took for him to get used to the idea of self-injecting:

"I said 'no chance, I'm not doing that'. I won't stick a needle- I can't look at people stick needles in me. [Nurse], she persuaded me to have a go at doing it, which we did. It took 3 or 4 months, maybe longer." (ID35 IV Home/M).

A couple of patients voiced concerns about how the product is made and that there might be a risk that they could pick up an illness. This particularly related to the fact that Ig treatment is a blood product and so worries about contamination and getting an illness such as HIV or AIDS were expressed, although this was generally accepted as a risk that was worth taking considering the benefits of the treatment. However, this in itself led to worries and anxieties about the possibility of the treatment supplies running out and/or the possibility of switching treatments:

"You know to a new patient, they are going to refuse it, because people they don't want a medicine that comes from blood, someone else. People are afraid... Yes, from someone else, you know like AIDS and things like that. So, you're shocked you know, oh no, no, no, this situation, I don't want it. But then you decide to take it because there are no other options" (ID 46 Iv Hospital/M).

\section{Dependence/life revolves around treatment}

Overall, most participants interviewed reported accepting the treatment as it made them feel better and being grateful that they had access to this treatment. However, there was a sense of powerlessness and lack of control over their treatment regimen. The fact that treatment was ongoing was viewed as being an emotional challenge that patients had to accept and manage:

"I thought it was a quick-fix thing. Obviously, what I didn't hear, which I've now got the grip of, 
it has been explained to me, that of course, it isn't is it? It's long-term, well it's for life basically" (ID04 IV Hospital/M).

"I have my moments, if I'm honest, when I think what would really happen if I stopped taking it, because it's still even though it's a million times better, there are still times where I just think I wish I didn't have to do this" (ID 02 IV hospital/ M).

Those going to hospital felt like they had less control over when they took their treatment.

A lot of this feeling of powerlessness and lack of control over their treatment related to the inflexibility of when they could take the treatment and also their dependence upon this to stay well for the rest of their lives. Some participants expressed a feeling of acceptance that they would need Ig treatment forever and this would never change. This feeling of lack of control, was also compounded by the need to be extra vigilant during the infusion sessions for any reactions when receiving this medication.

\section{Travel}

It was evident that the patients in our study had to invest considerable effort in order to have their Ig therapy. Another aspect of this related to travelling to clinic appointments, which this theme and its subtheme i) logistical factors, relates too - especially for those receiving Ig treatment in hospital. This was often described as a burden and inconvenient:

"I phoned them and said 'look, I've been driving around for 20 minutes, and I cannot find parking anywhere'. I said 'I've tried the road, I've tried inside. So if I don't actually turn up to this appointment, you know it's not lack of trying" (ID 47 IV Hospital/F).

\section{Logistical factors}

Logistical factors such as problems finding parking, travelling long distances, having to rely on others for transport or using public transport contributed to the treatment burden. Not having to travel for treatment was one of the reasons cited for preferring home Ig therapy compared to hospital treatment because, as one woman on IVIg treatment at hospital said: "You think 'I've got to put a whole day aside just for this...it's 25 miles" (ID23 IV Hospital/F). Similarly, another woman on the same treatment described having to set off really early for their treatment: "We had to be up for 6 o' clock this morning. Well, ready for 6am" (ID20 IV Hospital/F), and a male participant shared the challenges of getting to the hospital to undergo his IVIg treatment which involved: "Now, I'm catching like three buses to get here and it's taking a lot longer" (ID12 IV Hospital/M).

\section{Role functioning}

This theme describes the different ways that the participants role functioning was negatively affected by the Ig treatment. Role functioning refers to an individual's ability to exercise their day to day duties. This theme had two sub themes including i) looking after family, and ii) impact on work/education/school.

\section{Looking after family}

Some participants discussed treatment disrupting their ability to carry out and fulfil their roles in life as they would like, this could be either as a student, employee or carer/parent:

“And I've got a daughter who's 17 now. I mean she was a lot younger then and when you're in hospital, you're thinking how they are at home. You know, $X$ [husband] was working all the time and $\mathrm{X}$ [daughter] had to go between different grandparents and things" (ID40 SC Home/F).

"P: Because it were depressing me that much, you know I need to come all time so I just kept, and I were working as well. I had to keep having days off work and stuff like that, and it was getting me right down like. So I just thought 'stuff it', I weren't bothered, and I probably went a few months, I've done it a few times you know.

\section{I: Stop taking it?}

P: It's got me down that much like, and because when I start getting like that, that has an effect at home, you know what I mean with kids and everything and our lass. So I just stopped going. But then I started to like feel drained in myself and everything and get more chest infections and stuff. Anyway, I've always ended up coming back and that so (ID12 IV Hospital/M).”

\section{Impact on work/education/school}

The experiences of stopping work, taking time off work, adjusting working hours for appointments and treatment varied across participants depending on age, type of work/study and their employer but sometimes this could have had long-lasting effects on their lives where they felt unable to fulfil their life time goals:

\footnotetext{
"Well it was difficult because first being diagnosed and going into you can imagine like the critical years in your education, so like $15 / 16$, going on to 17 , and trying to do GCSE's and other things when I'd had periods of time for the two or three years before that out of school for investigation purposes. That had quite a massive impact really" (ID24 IV Home/M).
} 
The opportunity to be promoted or advance in careers was therefore seen as being restricted by the need for Ig treatment. Those on home SCIg treatment found that they needed to take less time out of work than when having treatment at the hospital and overall that it was less disruptive to the other roles they needed to fulfil, although they often still had to adapt their work lives or their personal lives as partner or spouse to accommodate their treatment, as one male participant on IVIg treatment at home shared:

"That is my greatest frustration and it frustrates me as well because of like future implications, because I don't think I- it's going to be a hell of a nightmare for me to progress within a company, and yes that's about it really" (ID13 IV Home/M).

\section{Financial consequences}

In the UK, the National Health Service (NHS) covers the direct cost of Ig treatment. Therefore, it was not surprising that this was the least reported aspect of Ig treatment burden for the patients interviewed. However, several indirect costs were identified through the interviews, which are encompassed within this theme and its three sub themes, including travel cost, insurance and loss of earnings.

Travel was cited as the main indirect cost of treatment for those on IVIg hospital treatment depending on the distance travelling and mode of transport, as highlighted by two male participants who explained: "So I virtually got 30 to 40 miles worth of petrol, plus $£ 3.70$ to park. So that's what the cost" (ID06 IV hospital/M), and "It is a pain to be fair, parking every time I come. It's not as bad now, but when I used to have the sub-cut, and I used to come to the hospital, it's like $£ 5$ every week" (ID02 IV Hospital/M).

Whilst travelling to hospital was considered expensive, travelling overseas for holidays and work was also hindered due to the inability to get travel insurance, as highlighted in one exemplar quote from a male participant who receives IVIg treatment in hospital:

"I've had opportunities to go over to America, working over there, and I'm like I've got to have treatment, and travel insurance and that, they're not going to cover the cost of this treatment. For me to pay for it, it's probably really, from a financial standpoint- it's just causing me a lot of problems really" (ID01 IV Hospital/M).

The relapsing nature of the condition and the necessity to have more treatment and hospital visits also impacted on work for many of the participants, with some describing how they were forced to leave work or feeling afraid that this may happen, as reported below by one female participant on SCIg at home and one male participant on IVIg in hospital:
"At the time, I was very career-minded. I got a really, I got a really good job at the local authority at - Council, and I was quite high up. But then at that time, I was getting worse and worse and worse and I was having time and time off work. And obviously you can't have, so I was forced to leave my work" (ID36 SC Home/F).

"It does bother me. It does because what they'll do is they'll sack me. That's it, that's the bottom line. That's the way they are. It's cost-cutting isn't it. I've been there so long. I earn the most money of my grade amongst about 8 or 9 of us. If they can see a chance, you're gone, and that's what they'll use to get rid of me. But it's not my fault, and I've tried to explain this to them" (ID04 IV Hospital/M).

The burden of treatment therefore created worries in relation to work and the ability to fulfil employment, and this was a cause for concern in terms of the long-term financial consequences for patients if they were unable to work or had to reduce the number of hours they worked.

\section{Discussion}

Our study aim was to explore PID patient's experiences of the burden of Ig treatment, including those receiving IVIg and SCIg, either in a home or hospital setting. To the best of our knowledge, this is the first study which has solely sought to explore in-depth the potential burden of Ig treatment for this patient group. To do this we adhered to the concept of burden of treatment such that we sought to explore where receiving Ig therapy may cause work and effort for the patient. In this study, Burden of Treatment (BoT) was defined as the consequences of receiving treatment (these may be medication, therapies, or other interventions). This concept also entailed the "work of being a patient" - everything the patient needs to do to treat and manage their illness, for example, undergoing tests and investigations, visiting doctors, adhering to treatment regimens, and making lifestyle changes. ${ }^{23}$

Whilst all the patients had experienced improvements in their health-related quality of life as a consequence of Ig treatment, they also describe areas of the treatment that are burdensome and impact upon a wide spectrum of life domains. These burdens particularly related to the amount of time, organization and planning that is needed. They also faced numerous physical, social, relationship, emotional, role, travelling, and financial challenges in their effort to undergo and maintain their infusions and care for their health. Our findings concurred with the results of the quantitative studies in this area. ${ }^{13-22} \mathrm{We}$ found the biggest burden of Ig therapy was in relation to time, with over two thirds of patients reporting this as a treatment burden.

Overall, the patients had experienced some mild sys- 
temic reactions to the therapy as expected, ${ }^{31}$ although a couple of patients had reported more extreme reactions to their treatment. Immediate systemic reactions with IVIg therapy are usually mild but include head and body aches, chills and fever. Allergic reactions are uncommon. ${ }^{6}$ Other more serious reactions can include headache, aseptic meningitis, kidney failure, blood clots and haemolytic reactions. ${ }^{32}$ There is also a risk of more variable blood levels of IgG compared to the subcutaneous route of administration. A low trough IgG level prior to intravenous infusion can result in patients experiencing symptoms of fatigue, general malaise and increased susceptibility to infection. ${ }^{32}$ Systemic adverse reactions to SCIg are also rare, however local adverse reactions such as persistent pain, bruising, swelling and erythema are common. ${ }^{31}$

In our study, there were different descriptions in the reports of treatment burden between the modes of treatment (SCIg and IVIg) and whether people had treatment at home or in hospital. A systematic literature review and a meta-analysis concluded that the efficacy of SCIg replacement was similar to IVIg treatment, however better treatment satisfaction, quality of life, and faster recovery (e.g. reduced time off work) was also observed. ${ }^{33}$

From the qualitative accounts of treatment that surfaced in our study, SCIg at home was associated with reduced travel, time and financial burden, greater comfort (both physically and in terms of being in a home environment) and less disruption to social activities.

Howard et al. ${ }^{14}$ reported that IV immunoglobulin treatment was burdensome for patients because of issues around scheduling therapy and travel. Gardulf et al. ${ }^{19}$ reported that patients on SCIg at home reported a significantly increased ability to participate in recreational activities. Similarly, Daly et al., ${ }^{13}$ found that patients undergoing home-based treatment (Sandoglobulin) had better Life Quality Index scores than clinic-based patients. Perceived benefits included greater convenience, comfort, independence, freedom to travel, flexibility of treatment schedule and a more pleasant treatment atmosphere. Home treated patients also reported less disruption of daily activities, waiting time and treatment-associated travel and cost.

However, whilst some of these benefits were experienced by the patients in our study, we also found there were areas where SCIg at home did cause a burden particularly in relation to the treatment environment (e.g. the need to have an infusion partner present during treatment, maintaining a clean and sterile environment in their homes, and lack of social contact that the hospital environment provided).

An infusion partner is someone close to the patient (e.g. a relative, a partner or friend) who is prepared to support the patient with their infusions. In the UK, the current consensus is that patients infusing via the subcutaneous route do not require an infusion partner, however decisions are made following an individual assessment. For example, some patients receiving subcutaneous infusions may still require an infusion partner e.g. due to lack of dexterity for using the equipment. If an infusion partner is identified then they are requested to attend a number of CIAU competency-based training sessions to enable them to carry out certain aspects of the infusion. These sessions, cover all aspects of the infusion, but focus on their role as the infusion partner. The aim is to have the training programme completed in 4-6 weeks however everyone is assessed individually and trained to meet their individual needs. The actual length of training will depend on the rate of learning. This person has to undergo a number of weeks of training to allow the person to infuse at home rather than in clinic. Thus, the level of support varies. In the UK, it is also not routine to actively carry out prehome visits. Instead, how and where infusions take place is typically discussed with their healthcare professional and the patient and partner.

Patients undergoing their Ig treatment at the CIAU felt that the clinical team took on these considerations and therefore they did not need to worry. It was notable that the staff at the clinic went out of their way to accommodate people for their needs and that overall people had very positive experiences at the clinics.

A couple of conceptual models have recently been proposed to describe burden of treatment. ${ }^{9,24}$ Although generated from a sample of patients with complex health conditions and therefore undergoing a variety of different medical treatment regimes, Eton et al. ${ }^{24}$ identified three broad themes; i) the work patients must do to care for their health, ii) problem focused strategies and tools to facilitate the work of self-care and iii) factors that exacerbate perceived treatment burden (i.e., challenges taking medication, emotional problems with family and friends, role and activity limitations, financial challenges, confusion about medical information, systemic obstacles of health care delivery. Similarly, Sav et al. ${ }^{9}$ presented four themes on the burden of treatment with chronic disease relating to financial burden, time and travel burden, medication burden and healthcare access burden.

In our study, these models were not used as a framework for the analysis because we adopted an inductive, data driven approach. However, our identified themes generally support the previous generic conceptual frameworks on burden of treatment previously mentioned. ${ }^{9,24}$ One notable difference to Sav et al.' ${ }^{9}$ findings was that we found concerns around financial issues to be the least burdensome. However, this different finding is likely because Sav et al.' ${ }^{9}$ research was conducted in Australia where the healthcare system is funded differently to the UK. Studies that have measured the burden of Ig treatment in a non-UK population have also found issues around costs and insurance to be a problem. ${ }^{14}$ Indeed, in the previous chronic condition literature, the healthcare system in which a treatment is given affects the perceived burden of the treatment. 
Another notable difference to Sav et al.'s ${ }^{9}$ findings were the emotional, relationship and social burden themes. Although generated from a sample of patients with complex health conditions and therefore undergoing a variety of different medical treatment regimes, Eton et al. ${ }^{34}$ reported that emotional problems with family and friends, role and activity limitations were some of the factors that could exacerbate perceived treatment burden. However, the psychological burden of the treatment was also largely underexplored. Also, in relation to the framework proposed by Eton et al. ${ }^{24}$ we only identified a couple of provider level issues but this may be because we recruited from one unit where the patients had very good relationships with their staff. The patients also didn't seem to be confused about any aspects of their Ig treatment.

To our knowledge this study provides the first in-depth qualitative study of patient's experiences of the burden of both IVIg and SCIg treatment for PID..$^{25}$ In focusing upon the work and effort that PID patients describe needing to undertake to receive this treatment, there may be some positive experiences of receiving this treatment that have not been reported or described by the patient. However, it is worth stating that despite the burdensome aspects of Ig treatment reported in this paper, there was an overwhelming sense of gratitude that Ig treatment is available to help manage their chronic condition which would not be possible without it. The sample size is modest but is expected for a qualitative piece of research and data saturation was reached. We recruited all the patients from one hospital in the UK. It may be that there are aspects of treatment burden that were not reported in this population, and patients in other sites or countries may have different experiences. This is particularly relevant in relation to the financial burden associated with Ig treatment as all the patients had their Ig treatment paid for by the NHS which is free at the point of treatment. The perceived level of burden upon leisure and social activities (in particular, holidays) described by some IVIg patients in this study may also be less for those being treated in other parts of the world. Local practice is not to switch between IVIg and SCIg products if possible. However, every effort is made to accommodate patients' holiday arrangements and arrange IVIg infusions (for patients on IVIg) around these. Whilst longer periods of travel has necessitated more planning, this has also been accommodated by liaising with local immunologists in the holiday destinations.

For patients on SCIg home therapy, local practice is that patients can take their product with them, so it should not interfere with holiday arrangements and letters for plane travel are also provided if required. As the patients on IVIg therapy described the treatment as causing more burden to their holidays, it would be interesting to explore this further, with the aim of identifying the ways this felt burden could potentially be reduced further in the local setting.

Our sample of patients included more patients who were undergoing IVIg treatment $(\mathrm{n}=21)$ compared to only nine patients who were receiving SCIg. The difference in sample numbers is because, at the time the study was conducted, the CIAU were treating more patients on IVIg therapy than SCIg. In addition, fewer home therapy patients returned the consent forms through the post. More IVIg patients were recruited because they were given their consent forms whilst attending for treatment at the clinic. This could mean that there's a bias towards those on IVIg hospital treatment in the results which may have occurred as a result of enhanced trust established while meeting the researchers. Although our reflexive thematic analysis illuminated patient perspectives, further research might use other methodologies, such as discourse analysis, to examine how patient accounts are [co] constructed in interview settings, thereby highlighting the interactional nature of interview data, and/or to analyse consultations between health care professionals and patients to explore how patient burden is negotiated in situ..$^{35}$

The choice of which route to use for Ig administration depends upon the personal preference of the patient, ease of intravenous access, tolerability of any previous Ig products, and the patient's lifestyle. It can be reviewed regularly and adjusted throughout the period of treatment as a patient's circumstances change. To further support patients in this decision-making process and improve patient-centred care plans, it may be beneficial for healthcare providers to share and discuss the potential work and effort for the patient that Ig treatment may result in. In turn, this may further help providers to deliver minimally disruptive medicine by tailoring treatment regimens to the realities of the daily lives of patients and relevant patient goals. ${ }^{36}$ Patients are likely to benefit from education to encourage them to acknowledge any felt treatment burden and talking with their health care provider about what can be possibly done to reduce it, which might foster adherence. ${ }^{37}$ It should also be noted that experts believe increased patient and physician shared decision-making (SDM) can result in better overall longitudinal care but understanding the physician's role in facilitating SDM is still limited, hence should be further explored. ${ }^{38}$

Most patients and healthcare providers are aware of treatment burden, however the lack of a simple method to measure it in the clinical setting often results in lack of focus on this important aspect of patient - centred care. As identified in a previous systematic review, many instruments are available to measure patient treatment burden, however there is no one standardized assessment method. To measure treatment burden, a few generic burden of treatment questionnaires are currently available, but these have been designed to capture treatment burdens across a wide range of chronic illnesses, for example, the Patient Experience with Treatment and Self-Management questionnaire. ${ }^{34}$ Some of the patient's perceived Ig treatment burdens we identified would be captured in this 
measure e.g. role and social activity limitations, however others would not in particular their described accounts of fear and worry about needles, catching another illness from the blood product, and other specific psychological and physical consequences associated with their Ig treatment. One of the key differences is that these generic questionnaires ask questions in relation to 'medications' but for the Ig group, their treatment is infusion based and so some of questions and the phrasing of these are not relevant to this PID cohort of patients.

As people live longer with chronic conditions there is an increased need for identification of patients as well as their caregivers burdened by specific treatment in order to engage in approaches to reduce treatment burden effectively. A standard and validated assessment method to measure patients' with PID and their caregivers' treatment burden in the clinical setting might not only enhance treatment but also allow for comparison of different approaches to reducing PID treatment burden and foster ongoing evaluation and monitoring of burden across patient populations and time. More importantly, given that the existing generic burden of treatment questionnaires do not appear to capture all of the Ig specific treatment-related burdens, we have used the themes identified from this qualitative work to inform the development of a new condition-specific Ig burden of treatment measure for this patient group (IgBoT-35) that is applicable across the different modes of administration and treatment types (IVIg and SCIg). ${ }^{39}$ This also follows good practice which recommends that data generated from qualitative research with the patient group of interest is used to develop the items in a patient-reported outcome measure to ensure its content validity. ${ }^{40}$

\section{Conclusions}

In this qualitative study, we have explored the experiences of undergoing life-long Ig treatment as described by patients diagnosed with a PID. In doing so, it has provided the first in-depth, rich account of Ig treatment burden and has shown that the therapy can impact upon many areas of life - particularly in relation to the amount of time the therapy necessitates for PID patients on IVIg and SCIg treatment. There appear to be some qualitative differences between patient's accounts and experiences of the burden of IVIg treatment compared to SCIg treatment but larger studies are needed to confirm this. For example, IVIg treatment in hospital was associated with more travel burden than SCIg at home. But SCIg necessitated more work and effort to keep a sterile and clean environment and thus required more planning and organisation in this regard. Choice over treatment should be informed by patient's clinical and lifestyle needs as well as familiarity with treatment regime. To facilitate this, our advice is that healthcare providers should also discuss with their patients the different ways that Ig treatment can be burdensome so the pa- tient's personal circumstances and preferences can be accommodated. Whilst healthcare delivery advocates the use of tools in routine care that can assess quality of life, we also recommend this is supplemented with a standardised treatment burden outcome measure such as the IgBoT-35 as part of their routine clinical practice as this can further facilitate the monitoring and delivery of more effective and personalised patient-centred care to their PID patients in the future.

\section{References}

1. Espanol T, Prevot J, Drabwell J, et al. Improving current immunoglobulin therapy for patients with primary immunodeficiency: quality of life and views on treatment. Patient Prefer Adherence 2014;8:621-9.

2. Mozaffari H, Pourpak Z, Pourseyed S, et al. Health-related quality of life in primary immune deficient patients. Iran J Allergy Asthma Immunol 2006;5:23-7.

3. PID UK. What are PIDs? The basics. Accessed: $18^{\text {th }}$ February 2021 Available from: http://www.piduk.org/whatarepids/ basics

4. Ataeinia B, Montazeri A, Tavakol M, et al. Measurement of Health-Related Quality of Life in Primary Antibody-Deficient Patients. Immunol Invest 2017;46:329-40.

5. Gardulf A, Nicolay U. Replacement IgG therapy and selftherapy at home improve the health-related quality of life in patients with primary antibody deficiencies. Curr Opin Allergy Clin Immunol 2006;6:434-42.

6. Jolles S, Orange JS, Gardulf A, et al. Current treatment options with immunoglobulin $\mathrm{G}$ for the individualization of care in patients with primary immunodeficiency disease. Clin Exp Immunol 2015;179:146-60.

7. Tran VT, Montori VM, Eton DT, et al. Development and description of measurement properties of an instrument to assess treatment burden among patients with multiple chronic conditions. BMC Medicine 2012;10:68.

8. May CR, Eton DT, Boehmer K, et al. Rethinking the patient: using Burden of Treatment Theory to understand the changing dynamics of illness. BMC Health Serv Res 2014;14:281.

9. Sav A, Kendall E, McMillan SS, et al. 'You say treatment, I say hard work': treatment burden among people with chronic illness and their carers in Australia. Health Soc Care Community 2013;21:665-74.

10. Mair FS, May CR. Thinking about the burden of treatment. BMJ 2014;349:g6680.

11. Brod M, Hammer M, Christensen T, et al. Understanding and assessing the impact of treatment in diabetes: the Treatment-Related Impact Measures for Diabetes and Devices (TRIM-Diabetes and TRIM-Diabetes Device). Health and Quality of Life Outcomes 2009;7:83.

12. Pifferi M, Bush A, Di Cicco M, et al. Health-related quality of life and unmet needs in patients with primary ciliary dyskinesia. Eur Respir J 2010;35:787-94.

13. Daly PB, Evans JH, Kobayashi RH, et al. Home-based immunoglobulin infusion therapy: quality of life and patient health perceptions. Ann Allergy 1991;67:504-10.

14. Howard V, Greene JM, Pahwa S, et al. The health status and quality of life of adults with X-linked agammaglobulinemia. Clin Immunol 2006;118:201-8.

15. Gardulf A, Bjorvell H, Andersen V, et al. Lifelong treatment 
with gammaglobulin for primary antibody deficiencies: the patients' experiences of subcutaneous self-infusions and home therapy. J Adv Nurs 1995;21:917-27.

16. Nicolay U, Haag S, Eichmann F, et al. Measuring treatment satisfaction in patients with primary immunodeficiency diseases receiving lifelong immunoglobulin replacement therapy. Qual Life Res 2005;14:1683-91.

17. Gardulf A, Nicolay U, Math D, et al. Children and adults with primary antibody deficiencies gain quality of life by subcutaneous IgG self-infusions at home. J Allergy Clin Immunol 2004;114:936-42.

18. Jones CA, Rojavin M, Baggish JS. Patients with primary immunodeficiency receiving subcutaneous immune globulin Hizentra maintain health-related quality of life and treatment satisfaction in a multicentre extension study of efficacy, tolerability and safety. J Pharmaceut Health Serv Res 2012;3: 41-7.

19. Gardulf A, Bjorvell H, Gustafson R, et al. The life situations of patients with primary antibody deficiency untreated or treated with subcutaneous gammaglobulin infusions. Clin Exp Immunol 1993;92:200-4.

20. Vultaggio A, Azzari C, Milito C, et al. Subcutaneous immunoglobulin replacement therapy in patients with primary immunodeficiency in routine clinical practice: the VISPO prospective multicenter study. Clin Drug Investig 2015;35: 179-85.

21. Gardulf A, Borte M, Ochs HD, Nicolay U. Prognostic factors for health-related quality of life in adults and children with primary antibody deficiencies receiving SCIG home therapy. Clin Immunol 2008;126:81-8.

22. Beauté J, Levy P, Millet V, et al. Economic evaluation of immunoglobulin replacement in patients with primary antibody deficiencies. Clin Exp Immunol 2010;160:240-5.

23. Jones GL, Vogt KS, Chambers D, et al. What is the burden of immunoglobulin replacement therapy in adult patients with primary immunodeficiencies? A Systematic Review. Front Immunol 2018;9:1308.

24. Eton DT, Ramalho de Oliveira D, Egginton JS, et al. Building a measurement framework of burden of treatment in complex patients with chronic conditions: a qualitative study. Patient Relat Outcome Meas 2012;3:39-49.

25. Petersson C, Fust R, Hagstedt C, et al. "Experiences of the burden of treatment"-Patient reports of facilitated subcutaneous immunoglobulin treatment in adults with immunodeficiency. J Clin Nurs 2018;27:4270-8.
26. Saunders B, Sim J, Kingstone T, et al. Saturation in qualitative research: exploring its conceptualization and operationalization. Quality Quantity 2018;52:1893-907.

27. Lincoln Y, Guba E. Naturalistic Inquiry. Newbury Park, CA: Sage Publications; 1985.

28. Braun V, Clarke V. Using thematic analysis in psychology. Qual Res Psychol 2006;3:77-101.

29. Vaismoradi M, Turunen H, Bondas T. Content analysis and thematic analysis: Implications for conducting a qualitative descriptive study. Nurs Health Sci 2013;15:398-405.

30. Friese S. Qualitative Data Analysis with ATLAS.ti: SAGE Publications Limited; 2019.

31. Stiehm ER. Adverse effects of human immunoglobulin therapy. Transfus Med Rev 2013;27:171-8.

32. Chapel H, Prevot J, Gaspar HB, et al. Primary immune deficiencies - principles of care. Front Immunol. 2014;5:627.

33. Abolhassani H, Sadaghiani MS, Aghamohammadi A, et al. Home-based subcutaneous immunoglobulin versus hospital-based intravenous immunoglobulin in treatment of primary antibody deficiencies: systematic review and meta analysis. J Clin Immunol 2012;32:1180-92.

34. Eton DT, Yost KJ, Lai JS, et al. Development and validation of the Patient Experience with Treatment and Self-management (PETS): a patient-reported measure of treatment burden. Qual Life Res 2017;26:489-503.

35. Potter J, Hepburn A. Qualitative interviews in psychology: problems and possibilities. Qual Res Psychol 2005;2:281-307.

36. May C, Montori VM, Mair FS. We need minimally disruptive medicine. BMJ 2009;339:b2803.

37. Sheehan OC, Leff B, Ritchie CS, et al. A systematic literature review of the assessment of treatment burden experienced by patients and their caregivers. BMC Geriatr 2019;19:262.

38. Lamb CC, Wang Y, Lyytinen K. Shared decision making: Does a physician's decision-making style affect patient participation in treatment choices for primary immunodeficiency? J Eval Clin Pract 2019;25:1102-10.

39. Jones GL, Williams K, Edmondson-Jones M, et al. The Development of a New Questionnaire to Measure the Burden of Immunoglobulin Treatment in Patients with Primary Immunodeficiencies: The IgBoT-35. Patient Prefer Adherence 2020;14:1567-84

40. Guidance for Industry Patient-Reported Outcome Measures: Use in Medical Product Development to Support Labeling Claims. Food and Drug Administration. U.S. Department of Health and Human Services; 2009. 